preschool children is significantly increased in the last decade (student t-test, p).

Conclusions Data from 2013 indicate that about 30\% of young children encounter the obesity problem. Therefore, children at this age already should represent the priority population for intervention strategies such as control of diet and/or physical activity.

\section{PO-0077 VITAMIN D DEFICIENCY IN CHILDREN WITH OSTEOGENESIS IMPERFECTA}

BL Meena, I Panigrahi, RK Marwaha. Pediatrics, Postgraduate Institute of Medical Education and Research (PGIMER), Chandigarh, India

10.1136/archdischild-2014-307384.747

Background and aims Osteogenesis imperfecta (OI) is a disorder that leads to fragile bones and significant morbidity. The aim was to find out the prevalence of Vitamin D deficiency in children with OI.

Methods In present study, 15 children with clinically severe OI on zoledronate therapy were studied. The biochemical parameters tested were Vit D level and urine DPD level along with the routine parameters like $\mathrm{Ca}$, Po, ALP, and urinary calcium creatinine ratio. We used a cut-off value of $30 \mathrm{ng} / \mathrm{ml}$ for vitamin D deficiency. Also cost effectiveness of zolendronate therapy was assessed.

Results Most of the OI patients were vit D deficient (80\%). The mean value of vitamin $\mathrm{D}$ in the study was $21.89 \pm 9.76$ (mean $\pm \mathrm{SD}$ ), and median value was 25.49 units. This treatment in present study did not significantly increase the financial burden on the family using alternate brand of zolendronate.

Conclusions High prevalence od Vitamin D deficiency in OI may be due to their less mobility and thus less sun exposure, the low vit $\mathrm{D}$ level can decrease their response to zolendronate treatment Vitamin D supplementation may be needed at higher doses along with oral calcium in patients with OI put on bisphosphonates therapy. Generic preparations of zoledronate do not increase the burden of therapy in patients with OI. Further studies are needed to find out long term side effects of zoledronate therapy in children.

\section{PO-0078 THE RELATIONSHIP BETWEEN MATERNAL AND NEONATAL 25(OH) VITAMIN D STATUS}

D Anuk Ince, A Ecevit, A Abbasoglu, U Tugcu, A Kurt, MA Tekindal, D Aldemir, A Tarcan. Department of Pediatrics, Baskent University School of Medicine, Ankara, Turkey

\subsection{6/archdischild-2014-307384.748}

Background In this study we aimed to investigate the relationship between maternal and neonatal 25(OH)D levels.

Methods The subjects were 58 mothers and their newborns who were born between February 2012 and April 2012. Blood specimens were obtained within $72 \mathrm{~h}$ of birth and from mothers. Serum 25(OH)D concentrations were measured. Vitamin D deficiency was defined as serum concentrations $\leq 20 \mathrm{ng} / \mathrm{mL}$.

Results The mean gestational age and birth weight of preterm infants were $33.06 \pm 2.2$ weeks and $2125.4 \pm 546 \mathrm{~g}$ and for term infants were $38.84 \pm 1$ weeks and $3470.3 \pm 451 \mathrm{~g}$, respectively. Sociodemographic characteristics of mothers were not significantly different between groups.
Twelve percent of infants born before 32 completed weeks, $16 \%$ infants born between 32-36 weeks and 28\% of term infants had vitamin D deficiency. Vitamin D deficiency was found in $27 \%$ mothers of preterm infants and $42 \%$ mothers of term infants.

Conclusion Vitamin D receptors plays an important role in calcium absorption and bone metabolism. In the literature there are reports that vitamin D deficiency during pregnancy had adverse gestational outcomes including risk of pre-eclampsia, gestational diabetes. The mean vitamin $\mathrm{D}$ levels were normal in infants whereas their mothers had low levels of vitamin D. When we consider that all mothers in the study received vitamin D supplements, we should give appropriate vitamin D prophylaxis during pregnancy. Also we should give adequate vitamin D supplementation to the infants without any delay.

\section{PO-0079 URINARY N-TELOPEPTIDE LEVELS ARE NOT ASSOCIATED WITH VITAMIN D STATUS IN HEALTHY CHILDREN}

${ }^{1} \mathrm{CA}$ Stoian, ${ }^{1} \mathrm{JK}$ Mah, ${ }^{2} \mathrm{AC}$ Chin, ${ }^{1} \mathrm{~A}$ Khan. ${ }^{1}$ Pediatrics, Alberta Children's Hospital, Calgary, Canada; ${ }^{2}$ Pathology and Laboratory Medicine, Calgary Laboratory Services, Calgary, Canada

\subsection{6/archdischild-2014-307384.749}

Background and aims Urinary levels of N-telopeptide (NTx) have been reported to be a sensitive and specific marker of bone resorption. This cross-sectional study determined the urinary levels of NTx among healthy children living in Calgary and explored their relationship with age, sex and vitamin D status.

Methods We included healthy children 2 to 13 years of age who presented to the Alberta Children's Hospital for elective surgery during a 12-month period. Data including the child's weight, height, age, gender, ethnicity, dietary intake, vitamin intake, and physical activity were collected. Urinary NTx levels were measured with a commercially (Wampole Laboratories, Princeton) available competitive-inhibition enzyme-linked immunosorbent assay.

Results Urinary NTx levels were available for 968 out of 1862 participants, of whom 605 (62.5\%) were boys. The mean urinary NTx/Creatinine ratio was $605.4 \mathrm{nmol} / \mathrm{mmol}$ (SD 264.8, range 200-2985.1). We found that mean urinary NTx/creatinine excretion was higher in the younger children (2-5 years) compared to subsequent ages. There was no significant difference in urinary NTx levels between children with suboptimal vitamin D status (serum 25-hydroxyvitamin $\mathrm{D}<80 \mathrm{nmol} / \mathrm{L}$ ) compared to those with optimal vitamin D status.

Conclusions Higher urinary NTx levels were measured in our healthy paediatric participants compared to what has been reported in healthy adults. In healthy children, urinary NTx levels may not be a useful marker of increased bone turnover in face of suboptimal vitamin D status. Future research is needed to determine the effect of suboptimal vitamin D status on bone health in children.

\section{PO-0080 LAXITAS GENERALISATA}

B Stanimirov. Pediatrics, Health Center, NOVI SAD, Serbia

10.1136/archdischild-2014-307384.750 
Introduction Renal connective tissue, characterised by the presence of loose ligaments throughout the body. This condition affects the joints throughout the body and is a generalised hypermobility, occurs in about $5 \%$ of the population and can be genetically conditioned.

Objective To examine the incidence of renal connective tissue and their representation by age and sex, the applied treatment and the need for hospitalisation.

Methods and materials Our target group were patients aged 1014 years, who are treated in the Department of Children Health Care, Health Centre Novi Sad in period (2011 to 2013), And used data from the Health patient records, on which was conducted retrospective-prospective study.

Results After examining the medical records, we came up with the following data: 463 (59.28\%) males and 764 (62.31) females, in which it was manifested weak connective tissue with various spectrum disorders and distinctive bluish whites.

Conclusion Poor connective tissue occurs before puberty and causes a wide spectrum of disorders: vision problems, curve the spine, frequent injuries of joints, constipation, prolapse of heart valves and other. There a way to cure, but rather to alleviate symptoms and to slow its progression, there are numerous events which may be unpleasant, and even that complicated.

\section{PO-0081 PITUITARY STALK INTERRUPTION SYNDROME: CLINICAL, RADIOLOGIC AND THERAPEUTIC PARTICULARITY}

${ }^{1} \mathrm{M}$ Tfifha, ${ }^{1} \mathrm{D}$ Dhahri, ${ }^{1} \mathrm{H}$ Ajmi, ${ }^{2} \mathrm{~K}$ Kadri, ${ }^{1} \mathrm{~S}$ Mabrouk, ${ }^{1} \mathrm{~J}$ Chemli, ${ }^{1} \mathrm{~N}$ Zouari, ${ }^{1} \mathrm{~S}$ Hassayoun, ${ }^{2} \mathrm{~K}$ Tlili, ${ }^{1} \mathrm{~S}$ Abroug. ${ }^{1}$ Pediatrics, University Hospital Sahloul, Sousse, Tunisia; ${ }^{2}$ Radiology, University Hospital Sahloul, Sousse, Tunisia

10.1136/archdischild-2014-307384.751

Background Patients with congenital hypopituitarism might have the classic triad of pituitary stalk interruption syndrome (PSIS), which consists of: an interrupted or thin pituitarys talk, an absent or ectopic posterior pituitary (EPP), and anterior pituitary hypoplasia or aplasia. The most remarkable clinical manifestations of patients with PSIS was growth retardation.

Objective To analyse the clinical, auxological and radiologic characteristics of the patients with PSIS to achieve better comprehension of this pathology.

Methods Data of patients with PSIS were retrospectively analysed for the clinical, laboratory and imaging features.

Results Five patients were included ( 4 girls and 1 boy). They are aged at the first clinical manifestation from 1 month to 3 years. The symptoms that led to the diagnosis were failure to thrive in four cases and polyuria-polydipsia syndrome in one case.

A complete growth hormone deficiency was confirmed in four cases, one was complicated with central hypothyroidism and one was accompanied by central adrenocortical hypofunction.

The last patient present only central diabetes insipidus.

Hypothalamo-pituitary MRI was performed in all of the patients showed one or more elements of the classic triad.

A causative mutation was studied in two patients. None HESX1 or LHX4 mutations was found.

Conclusion The PSIS is relatively frequent. The outcome is progressive evolution towards panhypopituitarism. The treatment consists in hormone replacement therapy.

\section{PO-0082 ELEVATED BLOOD PRESSURE IN EMIRATI ADOLESCENTS: ROLE OF OBESITY?}

${ }^{1} \mathrm{~S}$ Al Tandi, ${ }^{1} \mathrm{M}$ Al Aghbari, ${ }^{1} \mathrm{~N}$ Al Zaabi, ${ }^{1} \mathrm{~A}$ Al Marzoogie, ${ }^{1} \mathrm{~S}$ Al Hadraami, ${ }^{2} \mathrm{~S}$ Shah. ${ }^{1}$ Medical Student, College of Medicine and Health Sciences- United Arab Emirates University, Al-Ain, United Arab Emirates; ${ }^{2}$ Institute of Public Health, College of Medicine and Health Sciences- United Arab Emirates University, Al-Ain, United Arab Emirates

\subsection{6/archdischild-2014-307384.752}

Objectives Although obesity is fast increasing, there are few data on the prevalence of high-normal or elevated blood pressure in United Arab Emirates.

Methods We conducted a school-based survey of a representative sample of youth $(\mathrm{n}=1524)$ aged 12 to 18 years in Al Ain, UAE. BP measurements were made with a manual sphygmomanometer by trained nurses. Additional measures included height, weight, and abdominal circumference. BMI $\geq 85$ th and $\geq 95$ th percentiles were used to define overweight and obesity according to the 2000 CDC growth charts. The prevalence of 'high normal' and 'elevated' BP was assessed by comparing the subjects' SBP and DBP with age-, gender-, and height-specific 90th and 95th percentile reference values from the US National High Blood Pressure Education Program. Metabolic syndrome was defined using International Diabetes Federation guidelines.

Results A high proportion of Emirati adolescents (21\%) were obese compared to their non-local counterparts (16\%). Off the study adolescents $6 \%$ had elevated blood pressure. A high proportion $(19.1 \%)$ of obese children had elevated blood pressure compared to those who were overweight $(4.8 \%)$ and normal (2.1\%). Prevalence of metabolic syndrome was high (42\%) among obese compared to their counterparts with overweight (14\%) and normal weight (5\%).

Conclusion The present findings emphasise the importance of the prevention of obesity in order to prevent future cardiovascular related problem such as hypertension.

\section{PO-0083 RELATIONSHIP BETWEEN 25 HYDROXY VITAMIN D AND OBESITY IN 2-7 YEARS OLD CHILDREN REFERRED TO A PAEDIATRIC HOSPITAL IN IRAN}

S Mohammadian, R Mortezazadeh, H Zaeri, M Vakili. Pediatrics, Child and Neonatal Research Center GOUMS, Gorgan, Iran

\subsection{6/archdischild-2014-307384.753}

Background In observational studies vitamin D was one of the factors associated with obesity.

Aims Determine the association between BMI and serum level of vit $\mathrm{D}$ in children from

Taleghani hospital as outpatient in Iran.

Design This was a cross-sectional observational study on 215 children, 2 to 7 years old referred to hospital in winter 2013 .

Methods and material In cross sectional study, it was measured weight, height, waist circumference with identical instrument, Also determined BMI, Vitamin D level was performed on ELISA method.

Statistical analysis Vitamin D levels less than $20 \mathrm{nmol} / \mathrm{L}$ was considered as deficiency, $20-30 \mathrm{nmol} \backslash \mathrm{L}$ as inadequate and equal to or greater than $30 \mathrm{nmol} \backslash \mathrm{L}$ as sufficient.it was applied t-test, ANOVA, Pearson correlation coefficient at the significant level of 0.05 , data were analysed by SPSS.

Results 125 children were male and the rest were females 184 children had vitamin D deficiency and only 31 cases had adequate level. The prevalence of obesity and overweight was 